\title{
Investigation of Beam and Spin Dynamics for EDM Measurements at COSY
}

\author{
Marcel Rosenthal ${ }^{1,2}$ on behalf of the JEDI collaboration \\ 1. Institut für Kernphysik, Forschungszentrum Jülich, 52428 Jülich, Germany \\ 2. Physics Institute III B, RWTH Aachen, 52056 Aachen, Germany
}

Electric Dipole Moments (EDMs) are fundamental properties of elementary particles and are defined by a charge separation inside the particle. They violate both parity and time reversal symmetries. Assuming that the CPT theorem holds, this indicates CP violation. The known sources of CP violation described in the Standard Model of Particle Physics are not sufficient to explain the matter-antimatter asymmetry in universe. A direct measurement of a non-vanishing EDM would be a strong hint for physics beyond the Standard Model. So far, no direct measurements of charged hadron EDMs have been performed. The JEDI (Jülich Electric Dipole moment Investigations) collaboration investigates the feasibility of such measurements at storage rings [1]. Various methods are explored with respect to their statistical and systematical limits. The existing storage ring in Jülich, the Cooler Synchrotron COSY [2], is used to review these methods and to finally conduct a first direct measurement of proton and deuteron EDMs.

At COSY polarized protons and deuterons are produced in a particle source, pre-accelerated in a cyclotron and finally injected vertically polarized into the storage ring. There they can be further accelerated up to momenta of $3.7 \mathrm{GeV} / \mathrm{c}$. The JEDI test experiments currently performed utilize polarized deuterons with a momentum of $970 \mathrm{MeV} / \mathrm{c}$. The storage ring has a length of 183 meters and consists of 24 dipole magnets to guide and 56 quadrupole magnets to focus the beam. In the used lattice configuration the dispersion in the two straight sections of COSY is minimized and chromaticity corrections are applied using the three available sextupole magnet families in the arcs. Electron cooling is applied to reduce the transversal beam emittances and momentum spread. Furthermore an orbit diagnosis and correction system consisting of roughly 60 beam position monitors (BPMs) and 40 correction dipoles is used to monitor and to reduce orbit excitations. Polarization manipulation during particle storage is achieved by a radiofrequency ( $\mathrm{rf}$ ) solenoid and a recently developed $\mathrm{rf} \mathrm{E} \times \mathrm{B}$-dipole consisting of a superposition of electric and magnetic field in a Wien filter configuration.

In order to simulate the beam and spin dynamics and track particles in this lattice the simulation code COSY INFINITY [3] is utilized. It allows for computation of ring magnets' transfer maps to arbitrary order using differential algebra techniques. For the purpose of EDM studies the spin motion in homogenous electromagnetic fields in case of a non-vanishing EDM was introduced. Furthermore the ability to calculate time-dependent transfer maps, which is necessary to model the influence of radiofrequency fields on beam and spin motion, was implemented. Since the particle motion is perturbed by imperfections of the ring magnets, the existing misalignment commands for shifts, tilts and rotations can be superimposed to study randomized sets of magnet misalignments and the ability of the present orbit correction system to rectify them. For convenient control of different simulation settings, a $\mathrm{C}++$ framework was developed, which converts the requested tasks for calculation of ring optics or particle tracking into the COSY INFINITY script language and executes them. The results are further processed in the framework and later on stored in the ROOT [4] tree structure, which allows for fast, powerful and memory efficient post-processing and analysis. This framework was used to study different aspects of the proposed EDM measurement method, which are described in the following. 
The EDM $\overrightarrow{\mathrm{d}}$ is parallel to the spin $\overrightarrow{\mathrm{S}}$. The magnitude can be described by a dimensionless factor $\eta$ :

$$
\overrightarrow{\mathrm{d}}=\eta \cdot \frac{\mathrm{e}}{2 \mathrm{mc}} \overrightarrow{\mathrm{S}} .
$$

$\mathrm{e}$ and $\mathrm{m}$ denote the charge and the mass of the particle. For deuterons $\eta=10^{-5}$ corresponds to an EDM of roughly $\mathrm{d}=5 \cdot 10^{-20} \mathrm{e} \cdot \mathrm{cm}$. The spin motion of a particle passing homogenous electromagnetic fields is described by the Thomas-BMT equation [5,6] extended by the EDM contribution [7]:

$$
\begin{aligned}
& \frac{\mathrm{d} \overrightarrow{\mathrm{S}}}{\mathrm{dt}}=\overrightarrow{\mathrm{S}} \times \vec{\Omega}_{\mathrm{MDM}}+\overrightarrow{\mathrm{S}} \times \vec{\Omega}_{\mathrm{EDM}}, \\
& \vec{\Omega}_{\mathrm{MDM}}=\frac{\mathrm{e}}{\gamma \mathrm{m}}\left[\mathrm{G} \gamma \overrightarrow{\mathrm{B}}-\left(\mathrm{G} \gamma-\frac{\gamma}{\gamma^{2}-1}\right) \frac{\overrightarrow{\mathrm{E}} \times \vec{\beta}}{\mathrm{c}}-\frac{\mathrm{G} \gamma^{2}}{\gamma+1} \vec{\beta}(\vec{\beta} \cdot \overrightarrow{\mathrm{B}})\right], \\
& \vec{\Omega}_{\mathrm{EDM}}=\frac{\mathrm{e}}{\mathrm{m}} \frac{\eta}{2}\left[\frac{\overrightarrow{\mathrm{E}}}{\mathrm{c}}+\vec{\beta} \times \overrightarrow{\mathrm{B}}-\frac{\gamma}{\gamma+1} \vec{\beta}\left(\vec{\beta} \cdot \frac{\overrightarrow{\mathrm{E}}}{\mathrm{c}}\right)\right] .
\end{aligned}
$$

The spin vector $\vec{S}$ is given in the particle rest frame. The electric and magnetic fields $\mathrm{E}$ and $\mathrm{B}$ and the time $t$ are given in the laboratory system. $\beta$ and $\gamma$ denote the relativistic Lorentz factors and $G$ the anomalous magnetic moment. The particular form of Eq. (2) reflects the spin motion with respect to the momentum vector. In case of a perfect pure magnetic ring with vertical guiding fields and no EDM the so-called invariant spin axis points along the vertical direction. Spin components in the horizontal plane will precess around this axis. The number of spin revolutions per particle revolution with respect to the momentum vector is given by the spin tune

$$
v_{\mathrm{s}}=\mathrm{G} \gamma .
$$

In case of a non-vanishing EDM the invariant spin axis is slightly tilted towards the radial direction due to the motional electric field. The tilt can be described by an angle $\xi$ given by

$$
\tan \xi=\frac{\eta \beta}{2 G} \text {. }
$$

This also results in a tiny change of the spin tune. The proposed method for an EDM measurement is the resonant buildup of an EDM related polarization signal using a radiofrequency $\mathrm{E} \times \mathrm{B}$-dipole in a Wien filter configuration with a vertical magnetic field. In the ideal case the Lorentz force in this device vanishes and for $\vec{\beta} \perp \vec{E}$ also the EDM contribution of this device is zero and the spin precesses around the vertical axis. If the field oscillation frequency of the $\mathrm{E} \times \mathrm{B}$-dipole is now adjusted to the spin precession frequency in the ring, this leads to a polarization oscillation from an initially vertical spin into the horizontal plane (for more details see [8,9]). Figure 1 illustrates the resonant polarization buildup obtained in a simulation for different magnitudes of the EDM. In case of a non-vanishing EDM the slow net buildup is superimposed by a fast oscillation with the spin tune frequency $v_{\mathrm{s}} \cdot \mathrm{f}_{\mathrm{rev}}$. For a vanishing EDM the buildup is zero, since the spin precession axes of the storage ring alone and the $\mathrm{E} \times \mathrm{B}$-dipole itself are both vertical.

An important requirement for this method is the preservation of the polarization during the buildup process. According to Eq. (3) the precession speed of the spin is energy-dependent. (The tilt $\xi$ also leads to a tiny increase of the spin tune, which was neglected in this study.) Since the mean energies of the beam particles are different, the spin vectors of the ensemble will decohere over time and the polarization is lost. The linear contribution to energy spread is cancelled by bunching the beam using an rf cavity. For a bunched beam the average change of the revolution time for each particle, which can be expressed up to second order by Eq. (5), vanishes. The path-length change $\Delta \mathrm{L}$ can be divided into the contributions of betatron (x, y) and synchrotron motion, as seen in Eq. (6). 


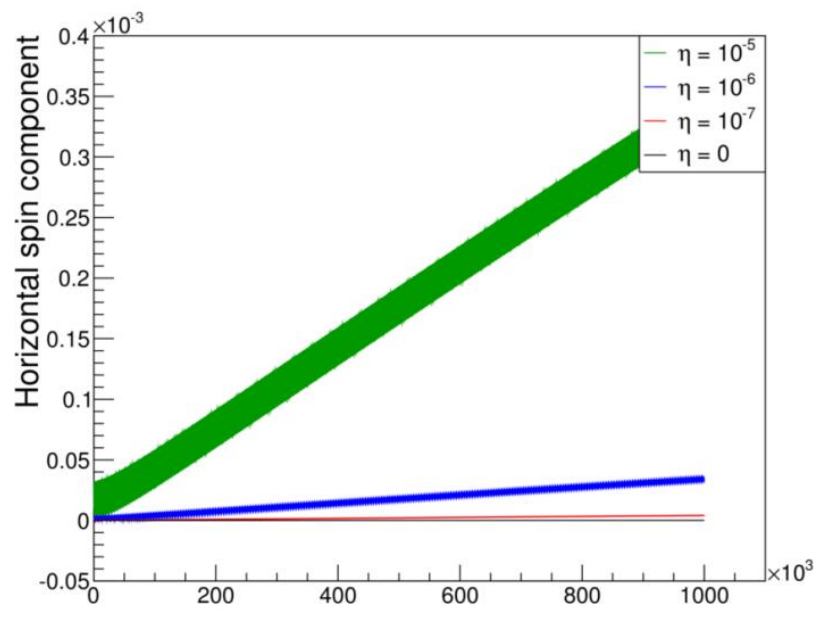

Figure 1. Simulated buildup of a horizontal spin component for a reference deuteron at $\mathrm{p}=970 \mathrm{MeV} / \mathrm{c}$ for different EDM magnitudes. The spin resonance was created by an $\mathrm{rf} \mathrm{E} \times \mathrm{B}$-dipole with vertical magnetic field of $0.3 \mathrm{mT}$ in a Wien filter configuration and a length of $0.6 \mathrm{~m}$.

$$
\begin{gathered}
\left\langle\frac{\Delta \mathrm{T}}{\mathrm{T}_{0}}\right\rangle=\left\langle\frac{\Delta \mathrm{L}}{\mathrm{L}_{0}}-\frac{\Delta \beta}{\beta_{0}}-\frac{\Delta \mathrm{L}}{\mathrm{L}_{0}} \frac{\Delta \beta}{\beta_{0}}+\left(\frac{\Delta \beta}{\beta_{0}}\right)^{2}\right\rangle=0, \\
\frac{\Delta \mathrm{L}}{\mathrm{L}_{0}}=\left(\frac{\Delta \mathrm{L}}{\mathrm{L}_{0}}\right)_{\mathrm{x}}+\left(\frac{\Delta \mathrm{L}}{\mathrm{L}_{0}}\right)_{\mathrm{y}}+\alpha_{0} \cdot \frac{\Delta \mathrm{p}}{\mathrm{p}_{0}}+\alpha_{1} \cdot\left(\frac{\Delta \mathrm{p}}{\mathrm{p}_{0}}\right)^{2} .
\end{gathered}
$$

For the synchrotron motion contribution the momentum compaction factor formalism up to second order is used, where p denotes the momentum. The combination of Eqs. (5) and (6) can be transformed into

$$
\left\langle\frac{\Delta \mathrm{T}}{\mathrm{T}_{0}}\right\rangle=\left(\alpha_{0}-\frac{1}{\gamma_{0}^{2}}\right)\left\langle\frac{\Delta \mathrm{p}}{\mathrm{p}_{0}}\right\rangle+\left(\alpha_{1}+\frac{3}{2} \frac{\beta_{0}^{2}}{\gamma_{0}^{2}}-\frac{\alpha_{0}}{\gamma_{0}^{2}}+\frac{1}{\gamma_{0}^{4}}\right)\left\langle\left(\frac{\Delta \mathrm{p}}{\mathrm{p}_{0}}\right)^{2}\right\rangle+\left\langle\left(\frac{\Delta \mathrm{L}}{\mathrm{L}_{0}}\right)_{\mathrm{x}}\right\rangle+\left\langle\left(\frac{\Delta \mathrm{L}}{\mathrm{L}_{0}}\right)_{\mathrm{y}}\right\rangle=0 .
$$

Replacing the average relative momentum change by the relative change of the Lorentz factor $\gamma$, one finds the three necessary conditions given in Eq. (8) to cancel the average energy spread:

$$
\begin{gathered}
\left\langle\left(\frac{\Delta \mathrm{L}}{\mathrm{L}_{0}}\right)_{\mathrm{u}}\right\rangle=-\frac{\pi}{\mathrm{L}_{0}} \cdot \epsilon_{\mathrm{u}} \cdot \xi_{\mathrm{u}}=0, \quad \mathrm{u} \in\{\mathrm{x}, \mathrm{y}\}, \\
\kappa \equiv\left[\alpha_{1}+\frac{3}{2 \gamma_{0}^{2}}\left(\beta_{0}^{2}-\left(\alpha_{0}-\frac{1}{\gamma_{0}^{2}}\right)\right)\right]=0 .
\end{gathered}
$$

The path-length change due to betatron motion can be expressed by the Courant Snyder Invariant of the phase space ellipse $\epsilon_{\mathrm{u}}$ (sometimes called single particle emittance) and the chromaticity of the accelerator in the corresponding direction $\xi_{\mathrm{u}}$, which describes the relative beam tune change with respect to a relative momentum change [10]. The parameter $\kappa$ has to vanish to cancel the path-length change due to second order momentum deviations. Both chromaticities and the second order momentum compaction factor $\alpha_{1}$ can be changed by the three sextupole families called MXS, MXL and MXG in the arcs of COSY. A simulation study was performed to validate the three conditions derived. Four different sextupole family configurations were chosen, where either one or all three conditions were fulfilled. A set of particles were randomly generated according to beam emittances of $5 \pi \mathrm{mm}$ mrad and an rms momentum spread of $5 \cdot 10^{-4}$ to test the so-called spin coherence time in the case that all 

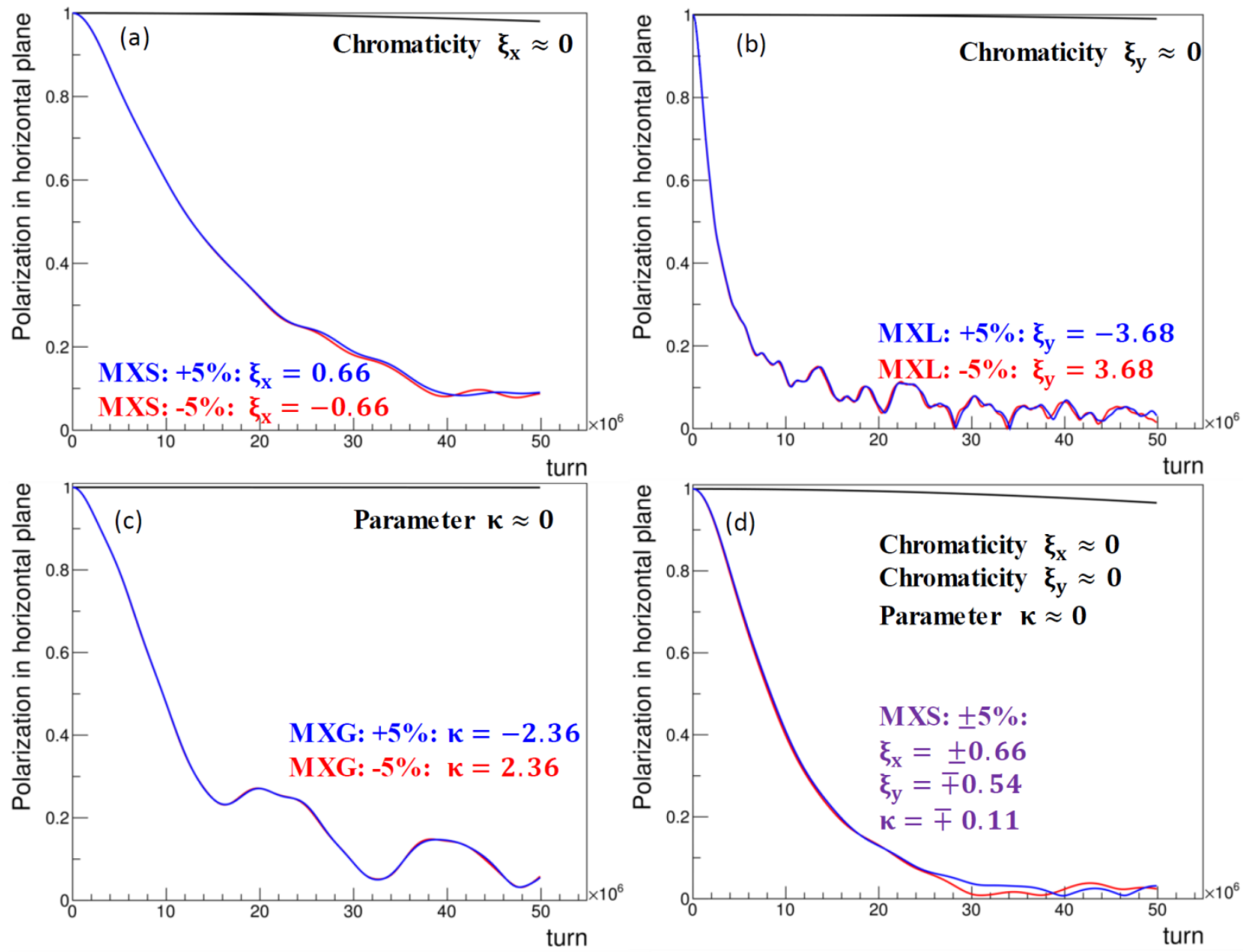

Figure 2. Simulated decoherence of the in-plane polarization due to various spin precession speeds in the particle bunch. Three sextupole families (MXS, MXL, MXG) were used to adjust the beam chromaticities $\xi_{u}$ and the parameter $\kappa$. In (a) the horizontal chromaticity and initial radially distributed particles, in (b) the vertical chromaticity and initial vertically distributed particles and in (c) the parameter $\kappa$ and initial longitudinally distributed particles were examined. In (d) all parameters were adjusted at the same time and the particles' initial coordinates were distributed in full phase space.

three conditions were fulfilled. For the configurations, which satisfied only one condition, only the corresponding 2D-phase space was populated, while the other four initial spatial coordinates were set to zero. The initial spin vectors of all particles were set along the longitudinal direction to probe for polarization loss. The tracking results of these ensembles are shown in Figure 2. For each combination of sextupole configuration and beam setup one sextupole family was varied additionally by five percent of its power supply maximal current to violate the required condition and illustrate the impact on the spin coherence time. The results of these simulations confirm the importance of the chromaticities and the parameter $\kappa$ for a long spin coherence time in case of deuterons with $\mathrm{p}=970 \mathrm{MeV} / \mathrm{c}$, which have a spin tune of $v_{\mathrm{s}} \approx-0.16$. The repolarization, which is visible in some of the graphs are an artefact of low statistics, but do not spoil the main purpose of this simulation. In general the radial fields of the focusing quadrupoles introduce an oscillating vertical spin component for particles which perform betatron oscillations. Combined with the precession around the vertical axis, this results in non-commuting spin rotations and a change of precession frequency. This effect is automatically included in the tracking 
simulations, but not in the previously described calculation for vanishing energy spread. For a perfect cancellation of second order spin decoherence effects the additional contribution to the spin tune spread has to be taken into account, especially for particles with larger anomalous magnetic moments.

Up to now only an ideal machine without imperfections was taken into account and the tilt of the invariant spin axis due to a non-vanishing EDM introduces an in-plane polarization buildup. Reconsidering Eq. (2) also radial and longitudinal magnetic fields, which occur due to misalignment errors and field imperfections of the ring magnets, may lead to a tilt of the invariant spin axis of the storage ring and can mimic the EDM effect. Since the anomalous magnetic moment is coupling to those fields, their impact is crucial for all considerations of EDM measurements at storage rings. A simulation was performed to quantify the influence of misalignments to beam and spin motion and the ability for correcting them with the present orbit correction system. For that purpose normally distributed misalignment errors of the dipole and quadrupole magnets of COSY were randomly generated. The standard deviation for shifts in radial, vertical and longitudinal direction were assumed to be $\sigma=0.1 \mathrm{~mm}$, respectively. Rotations around all three axes were randomized using a standard deviation of $\sigma=0.1 \mathrm{mrad}$. Furthermore, relative field errors of the bending dipoles in the order of $\sigma=10^{-4}$ were investigated. These imperfections introduce radial and vertical orbit excitations. An appropriate corrector setting was applied to correct for the orbit excitations at the BPM locations. In the shown studies the setup and measurement errors of the BPMs and correctors were not yet taken into account. A row of misalignment sets using different randomization seeds was generated. Before correction the excitations in both directions usually reach several millimeters and could be corrected to below $1 \mathrm{~mm}$ at most locations in this simulation. The change of spin tune due to these misalignments is shown in Figure 3. For the chosen standard deviations it reaches values around $\Delta v_{\mathrm{s}}=10^{-6}$, which could be reduced by one order of magnitude when applying orbit corrections. This change already indicates the impact on spin motion. In Figure 4 the polarization buildup using an $\mathrm{rf} \mathrm{E} \times \mathrm{B}$-dipole resonance method is shown, when either only imperfections or only an EDM is present in the simulations. For the assumed size of misalignments and applied orbit corrections the polarization buildup mimics an EDM in the order of $\mathrm{d}=10^{-19} \mathrm{e} \cdot \mathrm{cm}$. Without correction it can be even worse.

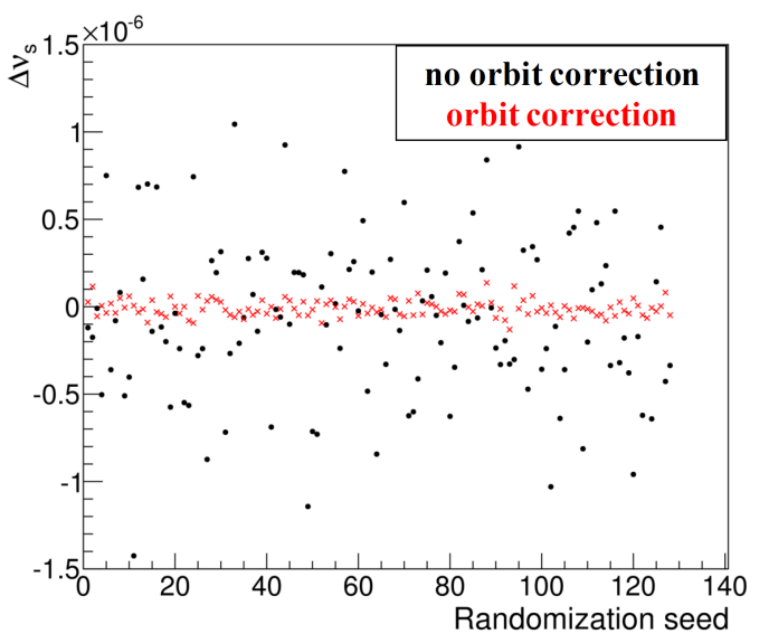

Figure 3. Simulated spin tune change due to randomized misalignments of dipoles and quadrupoles before and after an orbit correction using the existing BPM and correction dipole locations at COSY. The shifts and rotations were normally distributed with a standard deviation of $\sigma=0.1 \mathrm{~mm}$ and $\sigma=0.1 \mathrm{mrad}$, respectively. 

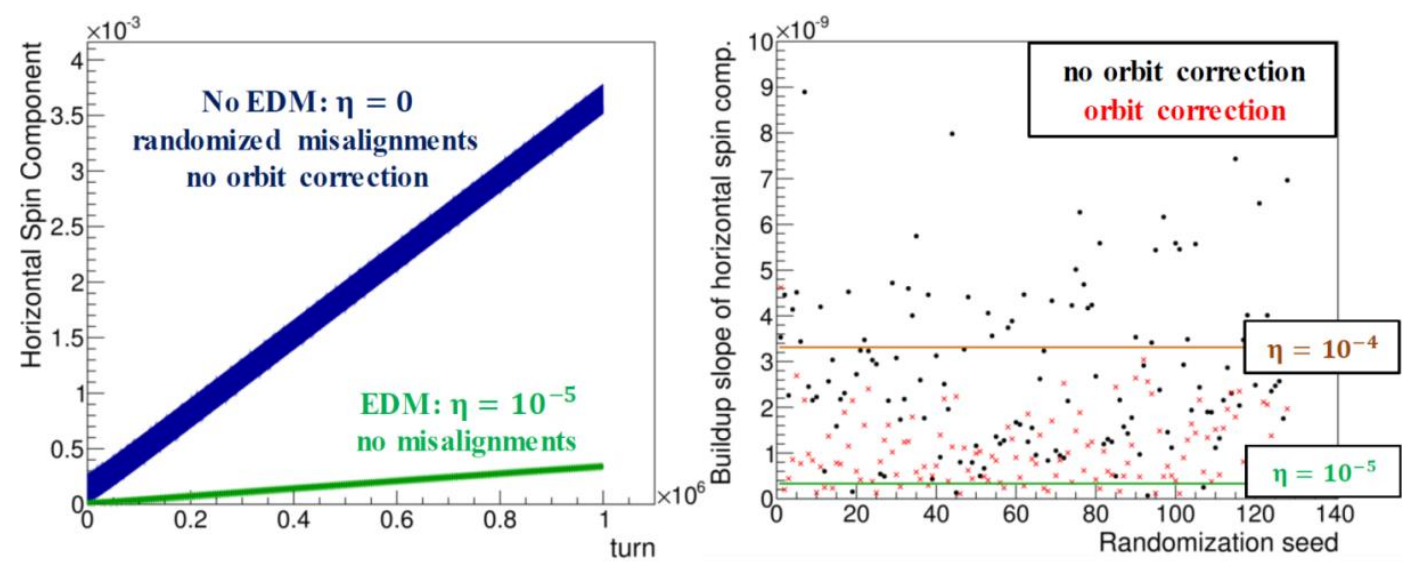

Figure 4. Simulated buildup of the horizontal spin component of a reference deuteron at $\mathrm{p}=970 \mathrm{MeV} / \mathrm{c}$. The spin resonance was created by an $\mathrm{rf} E \times \mathrm{B}$-dipole $(\mathrm{B}=0.3 \mathrm{mT}, 1=0.6 \mathrm{~m})$ in a Wien filter configuration. The left picture shows the buildup in case of a particular randomized dipole and quadrupole misalignment set compared to a non-vanishing EDM and no misalignments, respectively. The initial slopes were determined for different randomization seeds, which is illustrated in right panel. The points show the situation for misalignments, the straight lines mark the level of a pure EDM effect.

In these simulation studies the importance of beam chromaticities and momentum compaction factors on the spin coherence time for deuterons could be shown. In the case of deuterons with a momentum of 970 $\mathrm{MeV} / \mathrm{c}$ it was demonstrated that a long spin coherence time could be achieved by cancellation of the particles' energy spread inside the bunch. In a further step the development and implementation of timedependent transfer maps as well as the EDM extension to spin motion were tested and used to investigate the resonant polarization buildup method using an $\mathrm{rf} E \times \mathrm{B}$-dipole and its systematic limitations arising from the misalignments and field errors of the ring magnets. The presented limit is in the order of $\mathrm{d}=10^{-19} \mathrm{e} \cdot \mathrm{cm}$ for remaining orbit excitations below the millimeter level, which reveals the tough requirements for the proposed measurement in a pure magnetic ring [11].

\section{References:}

[1] A Lehrach et al in "Search for Permanent Electric Dipole Moments at COSY. Step 1: Spin coherence and systematic error studies", proposal to COSY PAC (2012/2014), http://collaborations.fz-juelich.de/ikp/jedi/

[2] R Maier et al, Nucl. Instrum. Meth. B 113 (1996), p. 26.

[3] K Makino and M Berz, Nucl. Instrum. Meth A 558 (2006), p. 346.

[4] R Brun and F Rademakers, Nucl. Insrumt. Meth. A 389 (1997), p. 81.

[5] LH Thomas, Phil. Mag. 3 (1927), p. 1.

[6] V Bargmann, L Michel and VL Telegdi, Phys. Rev. Lett. 2 (1959), p. 435.

[7] T Fukuyama and AJ Silenko, Int. J. Mod. Phys. A 28 (2013), 1350147.

[8] F Rathmann, A Saleev and NN Nikolaev, J. Phys. Conf. Ser. 447 (2013), 012011.

[9] W Morse, Y Orlov and Y Semertzidis, Phys. Rev. Spec. Top.-Ac. 16 (2013), 114001.

[10] Y Shoji, Phys. Rev. Spec. Top.-Ac 8 (2005), 094001.

[11] The author acknowledges the support of M. Berz and the whole MSU Beam Theory Group and the fruitful discussions within the JEDI group in Aachen and Jülich. 